\title{
Stress Hormone Response to Instrumented Lumbar Spine Fusion Surgery
}

\section{Jussi P. Repo ( $\nabla$ mrjussirepo@gmail.com )}

Department of Orthopaedic and Trauma Surgery, Tampere University Hospital, Tampere, Finland

\section{Marko Neva}

Department of Orthopaedic and Trauma Surgery, Tampere University Hospital, Tampere, Finland

\section{Keijo Häkkinen}

Biology of Physical activity, Faculty of Sport and Health Sciences, University of Jyväskylä, Finland

\section{Liisa Pekkanen}

Department of orthopedics and traumatology, Central Finland Health Care District, Jyväskylä, Finland.

\section{Saara Metso}

Department of Internal Medicine, Tampere University Hospital , Tampere, Finland

\section{Arja Häkkinen}

Health Sciences, Faculty of Sport and Health Sciences, University of Jyväskylä, Jyväskylä, Finland

\section{Research Article}

Keywords: Stress Hormone, Lumbar Spine Fusion

Posted Date: April 16th, 2021

DOl: https://doi.org/10.21203/rs.3.rs-418210/v1

License: (c) (i) This work is licensed under a Creative Commons Attribution 4.0 International License.

Read Full License 
2 Jussi P. Repo, MD, $\mathrm{PhD}^{1}$; Marko Neva, $\mathrm{MD}, \mathrm{PhD}^{1}$; Keijo Häkkinen, $\mathrm{PhD}^{2}$; Liisa Pekkanen, $3 \mathrm{MD}, \mathrm{PhD} ;{ }^{3}$ Saara Metso, $\mathrm{MD}, \mathrm{PhD}^{4}$, Arja Häkkinen, $\mathrm{PhD}^{5}$

4

Jussi P. Repo ORCID: 0000-0002-4688-5698 Tampere, Finland Jyväskylä, Finland Jyväskylä, Finland. Jyväskylä, Finland

Address for correspondence:

Tel. +358443593100
1. Department of Orthopaedic and Trauma Surgery, Tampere University Hospital,

2. Biology of Physical activity, Faculty of Sport and Health Sciences, University of

3. Department of orthopedics and traumatology, Central Finland Health Care District,

4. Department of Internal Medicine, Tampere University Hospital, Tampere, Finland .

5. Health Sciences, Faculty of Sport and Health Sciences, University of Jyväskylä,

Jussi P. Repo, Department of orthopedics and traumatology, Central Finland Health Care

District, Keskussairaalantie 19, 40620, Jyväskylä, Finland (e-mail: mrjussirepo@gmail.com)

19 
The purpose of the present study was to investigate stress, anabolic and catabolic hormonal levels and their association with interleukin 6 (IL-6) cytokine in patients undergoing lumbar spine fusion (LSF) surgery. Blood samples were collected preoperatively, and at 1, 3, 42 and 90 days postoperatively (POD) from 49 LSF patients with a mean (SD) age of 62 (11) years. Serum concentrations of adrenocorticotropic hormone (ACTH), cortisol, growth hormone $(\mathrm{GH})$, insulin-like growth factor 1 (IGF-1), testosterone, sex hormone-binding globulin (SHBG) and IL-6 were analyzed. In women, cortisol concentration rose above baseline values despite a fall in ACTH levels. GH showed a decrease on PODs 1 and 3 whereas IGF-1 levels remained stable. In males, SHBG increased, and both testosterone and free testosterone showed a decrease during PODs 1-3. The other hormone concentrations had returned to normal by PODs 42 or 90, except for IGF-1, which remained above the baseline value on PODs 42 and 90. IL-6 correlated significantly with cortisol $(\mathrm{p}<0.001)$ level on POD 1. The results suggest that hypercortisolism after operative stress is caused by cytokine-induced non-ACTH-driven cortisol production or reduced cortisol breakdown suppressing the production of ACTH via feedback inhibition. Furthermore, GH levels decrease rapidly. 


\section{Introduction}

The stress hormone cortisol is an essential component of the reaction to stress induced by surgical trauma. High cortisol levels during acute stress contribute to the supply of extra energy to vital organs by acutely shifting carbohydrate, fat, and protein metabolism and by delaying anabolism. Moreover, cortisol affects the hemodynamic system by initiating intravascular fluid retention and enhancing inotropic and vasopressor responses to catecholamines and angiotensin II. In addition, the anti-inflammatory effects of cortisol prevent over-activation of the inflammatory cascade. During acute stress, plasma cortisol concentrations are substantially elevated, a phenomenon that has traditionally been explained by the elevation of cortisol production in the adrenal cortex driven by adrenocorticotropic hormone (ACTH) secreted from the pituitary gland. However, evidence has accumulated showing that ACTH is elevated only transiently after acute stress (trauma, sepsis or critical illness) whereas cortisol concentrations remain high. ${ }^{1,2}$

Low plasma ACTH in the presence of high plasma cortisol concentrations has been interpreted as non-ACTH-driven cortisol production, a process in which cytokines could play a role. ${ }^{3}$ Alternatively, this phenomenon could be explained by reduced cortisol breakdown suppressing the production of ACTH via feedback inhibition. A study by Boonen et al. showed that cortisol production during a critical illness was only slightly higher than in healthy subjects. ${ }^{2}$ Cortisol breakdown was substantially reduced, probably explaining the increased levels of plasma cortisol despite no increase in its production. Both ACTH and cortisol have returned to their normal levels as early as 4-6 days after surgery in patients who have undergone hepatectomy. ${ }^{4}$ To our knowledge, no previous studies have investigated cortisol, ACTH and cytokine responses after acute surgical stress.

The main insulin counter-regulatory hormones are growth hormone, glucagon, adrenalin, noradrenalin and cortisol. Stress-induced endocrine responses ensure the adequate 
availability of glucose by activating gluconeogenesis, decreasing the insulin sensitivity of muscle cells and increasing the insulin sensitivity of organs predominantly relying on glucose, such as the brain and blood cells. ${ }^{5}$

Insulin-like growth factor 1 (IGF-1) is an endocrine mediator of growth hormone (GH)induced metabolic and anabolic actions, and it has paracrine and autocrine functions.

Circulating IGF-1 level is mainly controlled by pituitary GH secretion. Age, gender, smoking status and dietary intake have on effect on IGF-1 levels by affecting GH secretion and GH sensitivity. Cytokines and genetic factors may also influence circulating levels of IGF-1. In general, acute critical illness is characterized by an increase in circulating $\mathrm{GH}$ levels and a decrease in IGF-1 levels, indicating relative GH resistance. At least partially, this GH resistance is thought to be responsible for the negative nitrogen balance observed in a critical illness. ${ }^{6}$

Lumbar spine fusion (LFS) is performed by using an open midline approach in which the paraspinal muscles are detached from the spinous processes and retracted. Thereafter, the vertebrae are fixed together with transpedicular screws and rods and, if needed, with posterior lumbar interbody fusion is added. Perioperative tissue trauma undoubtedly causes an acute stress reaction that activates hormonal secretion. Acute stress affects several hormones; however, the mechanisms underlying these changes, and precisely when the changes occur, remain unknown. It is possible that an association exists between cytokine reaction and hormonal levels induced by surgical stress. Changes in serum hormonal levels caused by acute stress can impact on patient recovery and could explain the differences observed between individuals in their rates of recovery and complications. Moreover, the rise in cytokine levels in response to acute surgical trauma7 could also impact hormonal levels after surgery. ${ }^{8-10}$

This study investigated changes in serum cortisol, ACTH, GH and IGF-1 concentrations during the acute postoperative trauma and tissue repair process induced by instrumented LFS. In 
addition, changes in serum testosterone and sex hormone-binding globulin (SHBG) were investigated. A further aim was to assess the associations between interleukin (IL-6) cytokine and hormonal levels.

\section{Materials and methods}

\section{Ethical considerations and patient selection}

The ethics committee of Tampere University Hospital approved the protocol. All methods were performed in accordance with the relevant guidelines and regulations including the Declaration of Helsinki. Patients aged at least 18 years who provided a signed consent and for whom successful blood samples had been taken at the different follow-up time points were included. Patients diagnosed with inflammatory diseases or who had severe cardiovascular, psychiatric, social or general health disorders were excluded from the study. Patients who have hyper- or hypocortisolism, hypo- or hypergonadism, GH deficiency or pituitary gigantism would be excluded.

\section{Patients}

Altogether 52 consecutive patients with isthmic or degenerative spondylolisthesis or spinal stenosis who underwent an elective instrumented LFS in Tampere University Hospital were recruited for the study. The main indication for surgery was lower limb radicular pain. All operations were performed under general anesthesia in the operating theater. Glucocorticoid or immunomodulatory medication was not administered during the follow-up.

\section{Questionnaires}

Participants were asked to complete patient-reported outcome instruments and questionnaires on sociodemographic variables, duration of preoperative symptoms, and back and radicular pain at the time of admission. Information on patients' height, weight, medical conditions, 
current medication, tobacco use, and received treatments was also elicited by questionnaire.

Disability was captured by the Oswestry disability index (ODI). ${ }^{11,12}$

\section{Blood samples}

Patient blood samples were obtained pre- and postoperatively between April 2010 and November 2011 in Tampere University Hospital, Finland. All samples were collected in the morning before breakfast (before 7.30 a.m.) on the day of surgery, at 1 and 3 days postoperatively in the hospital ward, and at 6 weeks and 3 months postoperatively in a clinical follow-up at the hospital outpatient clinic. Blood was drawn from antebrachial venae into serum vacuum tubes. Centrifuged serum samples were stored at $-80 \mathrm{C}^{\circ}$ until assayed with an Immulite1000 analysator. Cortisol, ACTH, IGF-1, GH, testosterone, and SHBG levels were analyzed at each needed time point. Interleukin 6 (IL-6) levels were also analyzed at each time point. Free testosterone was calculated with Anderson's equation: serum free testosterone $(\mathrm{pmol} / \mathrm{l})=$ serum testosterone $(\mathrm{nmol} / \mathrm{l}) \times(2.28-1.38 \times \log (\operatorname{SHBG}(\mathrm{nmol} / \mathrm{l}) / 10)) \times 10$.

\section{Statistics}

Data are presented as means with standard deviations $(S D)$, medians with interquartile ranges (IQR), $95 \%$ confidence intervals $(95 \% \mathrm{CI})$ or as counts with percentages. Repeated measures for continuous outcomes (hormones) were analyzed using a generalized estimating equations (GEE) model with an unstructured correlation structure. The Friedman Test or Wilcoxon signed-rank test with post hoc Bonferroni correction was used for analyzing significant differences in hormone levels between each time point or group. Statistical significance was calculated using a permutation test or Fisher's exact test, or Sidak-adjusted probabilities for baseline differences and IL-6 levels, respectively. Spearman's correlation was used to obtain 
information on the associations of hormonal level with age, gender and time from surgery. Correlations between hormonal levels and cytokine IL-6 were also calculated. Analyses were performed using STATA 14.1 or SPSS 24.0.

\section{Results}

Blood samples were collected successfully from 49 of the 52 patients: 38 with degenerative spondylolisthesis and/or spinal stenosis and 11 with isthmic spondylolisthesis. Patients' mean age was 62.2 years (range, 49-93). Thirteen patients were male and 36 female. Fusion length was one or two levels in 35 patients and more than two levels in 14 patients. In addition, posterior lumbar interbody fusion was used in 12 patients. No postoperative infections were observed in the study group. Further sociodemographic and clinical details are described in Table 1. No significant gender differences were found in baseline characteristics.

\section{Hormone levels}

Serum cortisol concentrations had risen above the baseline level on POD 3 in women (Figure 1) but had returned to their baseline values at 6 weeks after surgery. The total variance was statistically significant $(p=0.04)$. However, a significant decrease in ACTH was noted already on PODs $1(p<0.001)$ and $3(p=0.0001)$ but not on PODs 42 and 90 .

In both sexes, GH decreased during the first three PODs but had returned to its baseline value by POD 42. IGF-1 decreased slightly during the first 3 PODs. IGF-1 had risen significantly above the preoperative level at 6 weeks and 3 months after surgery.

In men, serum SHBG concentration increased during the first 3 PODs $(\mathrm{p}<0.001)$.

Furthermore, serum testosterone showed a decrease on PODs 1 and POD 3 but had returned to normal by POD $42(\mathrm{p}<0.001)$. SHBG had decreased to its baseline level by 6 weeks after 
surgery. Free testosterone calculated by the Anderson's equation decreased significantly during the PODs $1-3$ but had returned to normal by 6 weeks after surgery $(\mathrm{p}<0.001)$ (Figure 2$)$.

\section{Correlation between IL-6 and hormones}

IL-6 was significantly elevated on PODs 1 and 3 (Figure 3) and showed a significant correlation with cortisol ( $\mathrm{p}<0.001$ ) levels on POD 1 (Table 2). No other statistically significant associations were found between IL-6 and hormonal changes on PODs 1 and 3.

\section{Discussion}

The present study showed marked changes in serum hormone concentrations after instrumented lumbar spine fusion. On POD 3, cortisol concentration had risen above, whereas ACTH fallen below, their baseline levels. The results do not support the theory that ACTH induces an increase in the secretion of cortisol in acute trauma. On the contrary, this result suggests that hypercortisolism is caused by cytokine-induced non-ACTH driven cortisol production or reduced cortisol breakdown, suppressing the production of ACTH via feedback inhibition. These results are in line with those of Boone et al. ${ }^{2}$ in critically ill patients. Both cortisol and ACTH had returned to their normal levels by 6 weeks after surgery. To the best of the authors' knowledge, post-surgical cortisol and ACTH levels have not previously been monitored for as long a period as in the present study.

GH and IGF-I have complex anabolic effects and are important regulators of muscle remodeling. ${ }^{13}$ Cortisol and anti-diuretic hormone levels have been shown to increase during Lumbar spine surgery and remain elevated at one-hour post-surgery. ${ }^{14}$ In the present study, with the exception of IGF-1, which continued to show an increase at 3 months after surgery, 
GH had returned to the preoperative level by 6 weeks after surgery. The increase in IGF-1 at 6 weeks and at 3 months could indicate a late anabolic effect after surgical trauma. Further studies would be needed to unravel the effect of IGF-1 on anabolic metabolism. IGF-1 levels are associated with age and gender, although to a lesser degree after age 40 . In the present study, no gender differences were observed in the baseline levels of IGF-1. The authors used IGF-1, as all the patients were at an age where the IGF-1 reference values do not change drastically by gender.

The results of the present study suggest that acute surgical stress has a dramatic impact on testosterone levels. Testosterone and SHBG levels should not be measured immediately after an operation, as the surgery can distort the levels of testosterone and SHBG. Free testosterone levels had returned to their preoperative level by 6 weeks after surgery. Hohman et al. investigated the effects of Nandrolone decanoate on recovery and muscle strength after total knee arthroplasty. ${ }^{15}$ Their randomized controlled study found that the use of anabolic steroids led to faster recovery and better muscle strength compared to controls. ${ }^{15}$ The stressrelated decrease in free testosterone is of physiologic origin. The decrease helps to transform muscles from the anabolic to catabolic state, thereby directing energy metabolism to the maintenance of critical functions.

Interleukin-6 (IL-6) is a proinflammatory cytokine that is activated by acute surgical trauma. IL-6 could be associated with acute stress hormones. ${ }^{8-10}$ To the best of the authors' knowledge, no previous studies have assessed the relationship between acute phase hormones and IL-6 levels. The results indicate that there might be a postoperative association between IL-6 and cortisol. No association with the other hormones studied, such as GH and testosterone, was found. Further studies with larger cohorts are needed to corroborate this finding. 

time points. The blood samples were obtained in real clinical settings both for ethical reasons and to ensure the minimum inconvenience to patients. The length of follow-up and intervals

205

between measurements can be considered adequate and compare favorably with those

reported in previous published studies. ${ }^{9-10,16}$ The study contained more women than men simply because the prospective unselective sample contained more women. A weakness of the study was that the comparison of testosterone levels in older patients is difficult, as testosterone levels are notably higher in men than in women. Furthermore, the study did not test luteinizing hormone levels. In the same way as the present analysis provided information on the function of the hypothalamus-pituitary-adrenal axis in the context of stress, the testing of luteinizing hormone might have yielded information on the mechanism governing changes in testosterone and SHBG. Future studies should use samples containing equal numbers of men and women and also stratified by age.

\section{Conclusions}

The results showed that ACTH, cortisol, IL-6, testosterone GH, and SHGB levels respond rapidly to tissue trauma. Surgical stress after instrumented lumbar spine fusion induces tissue trauma that elevates IL-6. Postoperative high cortisol was not associated with the elevation in the level of ACTH. The late elevation of IGF-1 seems to be associated with late tissue repair process. Further studies could investigate whether inter-individual differences exist in hormonal responses to surgical stress and whether surgical stress affects surgical outcomes or complications.

Conflicts of Interests: None to declare. 
225 Funding: The study was funded by the Academy of Finland (ref nro 133336) and by the

226 Competitive State Financing of the Expert Responsibility Area of Tampere University

227 Hospital.

228 Contributions

$229 \mathrm{AH}, \mathrm{KH}, \mathrm{MN}$ designed the study

230 JR (and HK) conducted the statistical analysis

231 JR and SM prepared the manuscript draft

232 All authors critically reviewed the final manuscript draft

233

234 Additional information

235 The authors report no conflicts of interest

236

237 Acknowledgements

238 The authors would like to thank Hannu Kautiainen for statistical consultation. The study is

239 funded by the Competitive State Financing of the Expert Responsibility Area of Tampere

240 University Hospital. 
244 Figure 1. Change in hormonal levels at postoperative time points in men and women.

245 Preoperative value has been set to zero (preoperative values are presented in Table 1).

246 Whiskers show 95\% CIs.

247 Figure 2. Change in free testosterone calculated using the Anderson's equation.

248 Figure 3. Change in IL-6 values at postoperative time points. Preoperative IL-6 value has been 249 set to zero. Whiskers show $95 \%$ CIs. A statistically significant difference $(p<0.001)$ was found 250 at all time points. 
Figures
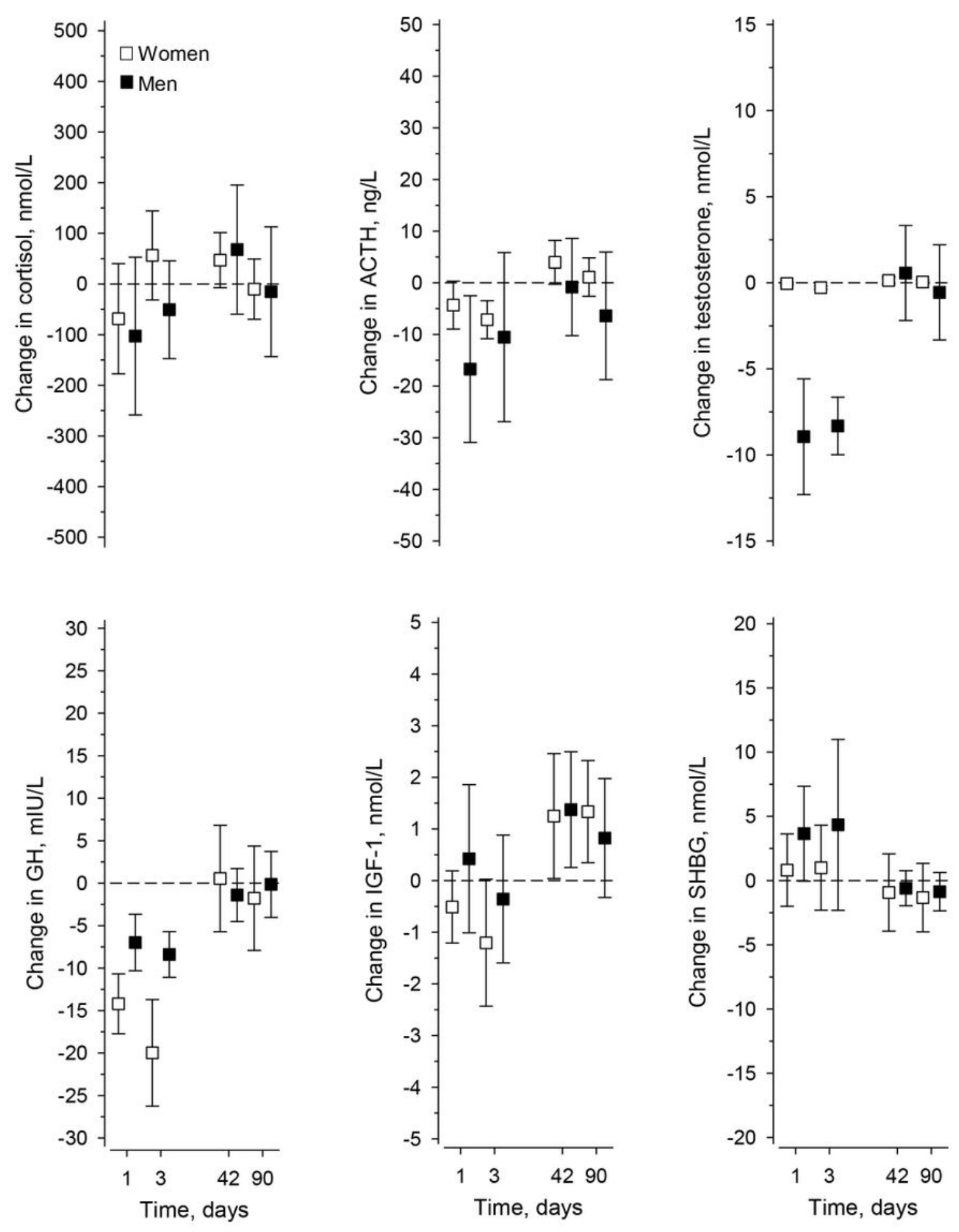

Postoperative days

\section{Figure 1}

Change in hormonal levels at postoperative time points in men and women. Preoperative value has been set to zero (preoperative values are presented in Table 1). Whiskers show 95\% Cls. 


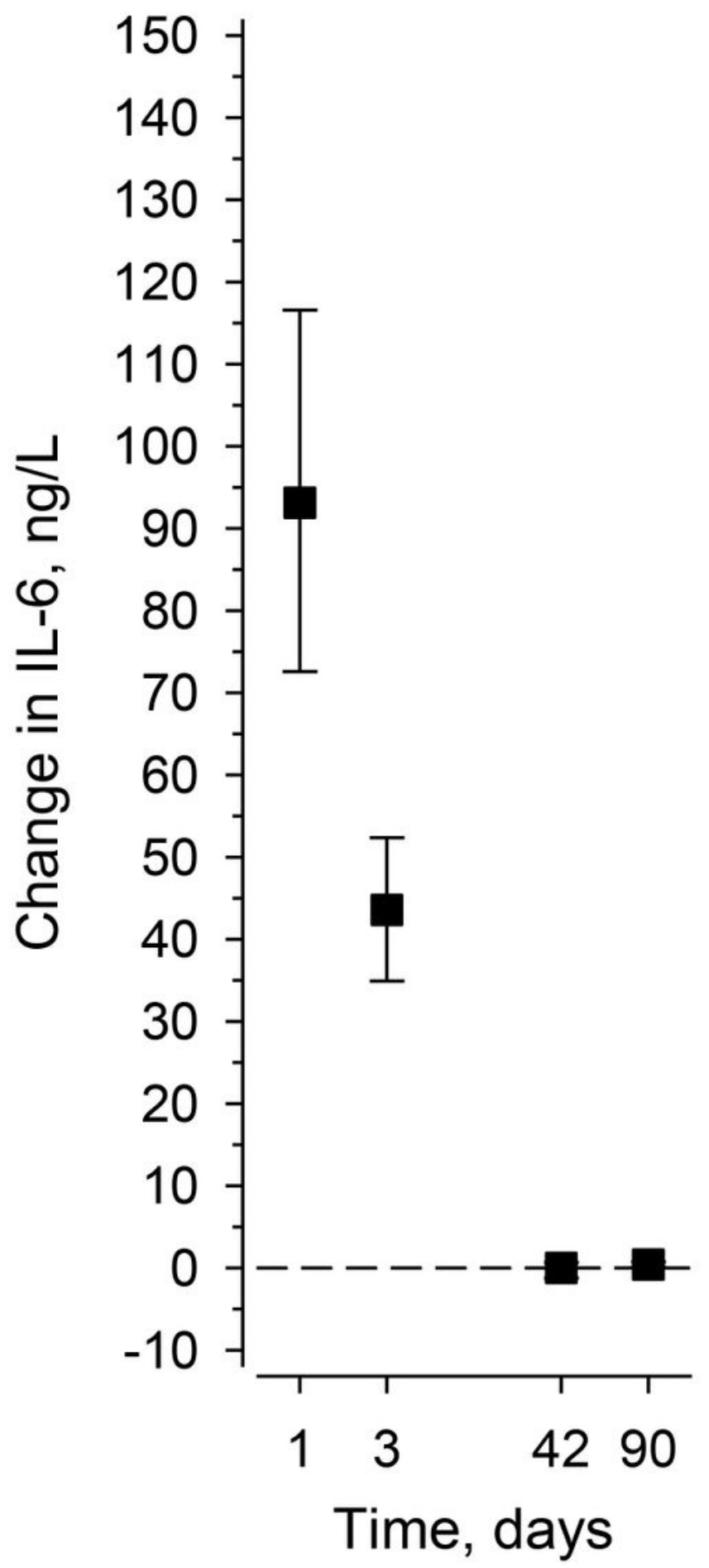

Figure 2

Change in free testosterone calculated using the Anderson's equation. 


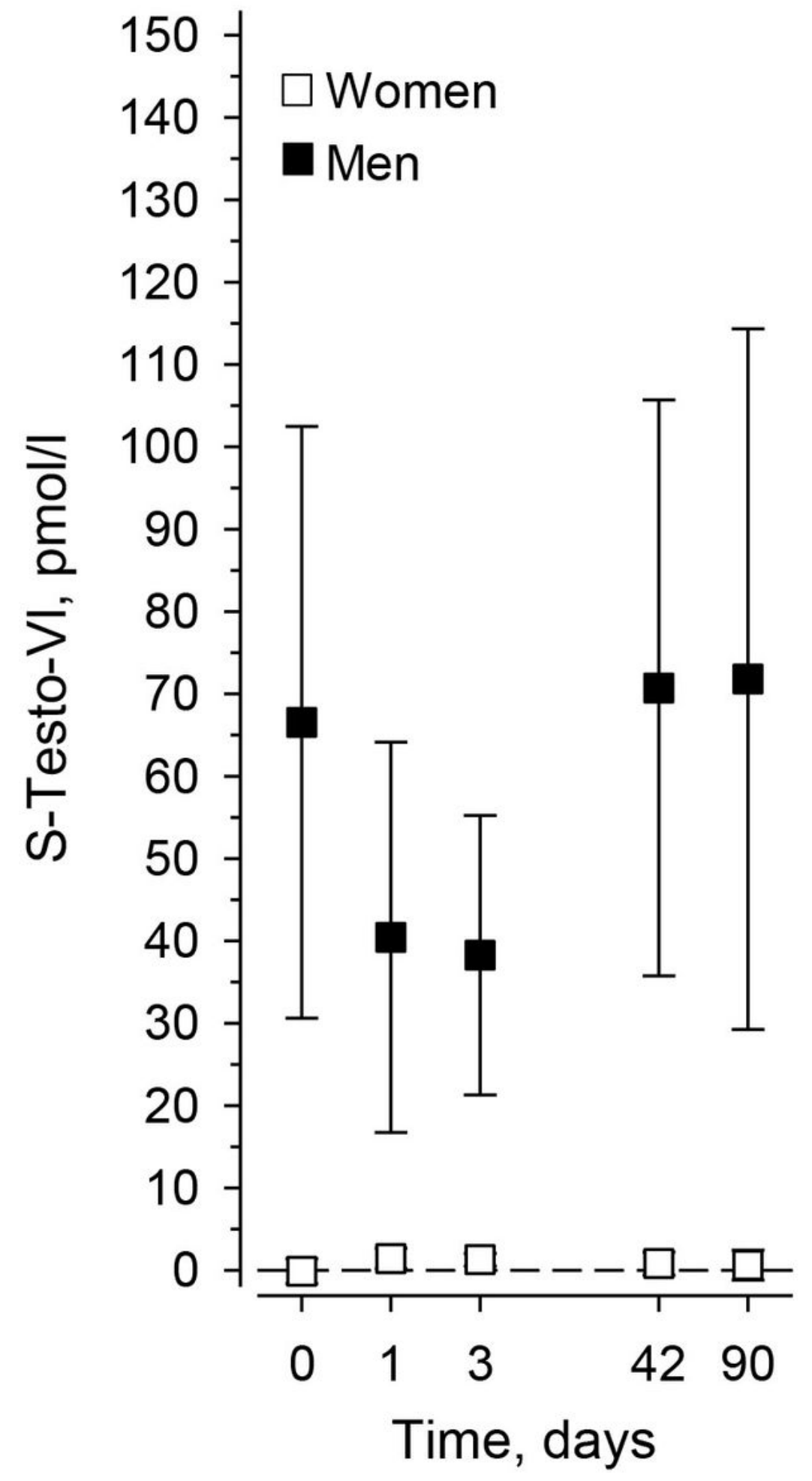

Figure 3

Change in IL- 6 values at postoperative time points. Preoperative IL- 6 value has been set to zero. Whiskers show $95 \%$ Cls. A statistically significant difference $(p<0.001)$ was found at all time points.

\section{Supplementary Files}


This is a list of supplementary files associated with this preprint. Click to download.

- Table1.pdf 